\title{
Extremely Low Forces Induce Extreme Axon Growth
}

\author{
Sara De Vincentiis, ${ }^{1 *}$ Alessandro Falconieri, ${ }^{1 *}$ Marco Mainardi, ${ }^{2,3}{ }^{\circledR}$ Valentina Cappello, ${ }^{4}$ Vincenzo Scribano, ${ }^{1}$ \\ Ranieri Bizzarri, ${ }^{5,6}$ Barbara Storti, ${ }^{5,6}$ Luciana Dente, ${ }^{1}$ Mario Costa, ${ }^{2,3}$ and ${ }^{-}$Vittoria Raffa ${ }^{1}$ \\ ${ }^{1}$ Department of Biology, Università di Pisa, Pisa 56127, Italy, ${ }^{2}$ Institute of Neuroscience, Consiglio Nazionale delle Ricerche (CNR), Pisa 56124, \\ Italy, ${ }^{3}$ Bio@SNS, Scuola Normale Superiore, Pisa 56127, Italy, ${ }^{4}$ Center for Nanotechnology Innovation (CNI), Istituto Italiano di Tecnologia, Pisa \\ 56127, Italy, ${ }^{5}$ National Enterprise for nanoScience and nanoTechnology (NEST), Scuola Normale Superiore and Institute of Neuroscience (CNR), \\ Pisa 56127, Italy, and ${ }^{6}$ Department of Surgical, Medical and Molecular Pathology, and Critical Care Medicine, Università di Pisa, Pisa 56126, Italy
}

Stretch-growth has been defined as a process that extends axons via the application of mechanical forces. In the present article, we used a protocol based on magnetic nanoparticles (NPs) for labeling the entire axon tract of hippocampal neurons, and an external magnetic field gradient to generate a dragging force. We found that the application of forces below $10 \mathrm{pN}$ induces growth at a rate of $0.66 \pm 0.02 \mu \mathrm{m} \mathrm{h}^{-1} \mathrm{pN}^{-1}$. Calcium imaging confirmed the strong increase in elongation rate, in comparison with the condition of tip-growth. Enhanced growth in stretched axons was also accompanied by endoplasmic reticulum (ER) accumulation and, accordingly, it was blocked by an inhibition of translation. Stretch-growth was also found to stimulate axonal branching, glutamatergic synaptic transmission, and neuronal excitability. Moreover, stretched axons showed increased microtubule (MT) density and MT assembly was key to sustaining stretch-growth, suggesting a possible role of tensile forces in MT translocation/assembly. Additionally, our data showed that stretched axons do not respond to BDNF signaling, suggesting interference between the two pathways. As these extremely low mechanical forces are physiologically relevant, stretch-growth could be an important endogenous mechanism of axon growth, with a potential for designing novel strategies for axonal regrowth.

Key words: axon growth; force; mechanotransduction

Significance Statement

Axon growth involves motion, and motion is driven by forces. The growth cone (GC) itself can generate very low intracellular forces by inducing a drastic cytoskeleton remodeling, in response to signaling molecules. Here, we investigated the key role of intracellular force as an endogenous regulator of axon outgrowth, which it has been neglected for decades because of the lack of methodologies to investigate the topic. Our results indicate a critical role of force in promoting axon growth by facilitating microtubule (MT) polymerization.

\section{Introduction}

The growth cone (GC) is a highly motile structure that enables the machinery to move forward. It also enables axon guidance by detecting guidance cues and translating them into directional movement. Since all these processes are driven by forces, it is not surprising that neurons sense and respond to mechanical forces. Today, it is widely accepted that mechanical force promotes axon growth, the process referred to as "stretch-

Received Dec. 31, 2019; revised Apr. 16, 2020; accepted Apr. 21, 2020.

Author contributions: M.M., V.C., R.B., M.C., and V.R. designed research; S.D.V., A.F., M.M., V.C., V.S., V.R.,

R.B., and B.S. performed research; S.D.V., A.F., M.M., and V.R. analyzed data; M.M., V.C., R.B., L.D., M.C., and

V.R. wrote the paper.

*S.D.V. and A.F. contributed equally to this work.

This work was supported by the Wings for Life Foundation. We thank Prof. Gerardo Goya for his helpful advice in relation to nanomaterials and magnetic field applicator.

The authors declare no competing financial interests.

Correspondence should be addressed to Vittoria Raffa at vittoria.raffa@unipi.it.

https://doi.org/10.1523/JNEUROSCI.3075-19.2020

Copyright $\odot 2020$ the authors growth" (O’Toole et al., 2008; Smith, 2009; Franze and Guck, 2010; Suter and Miller, 2011).

Experiments performed in the last two decades have highlighted that mechanical force regulates neurite elongation and sprouting (Suter and Miller, 2011) and nervous system development (Koser et al., 2016). Force can induce mass addition even when applied at sites other than the GC (Ruthel and Hollenbeck, 2000; Lamoureux et al., 2010). In fact, axon growth is regulated by cytoskeletal dynamics, which in turn are influenced by the strong contractile forces generated in the GC and the weaker contractile forces along the axon (Miller and Suter, 2018). Exogenously applied force can modulate these force gradients, acting as a powerful modulator of axonal growth.

In this context, the GC acts like a fine guidance sensor and can transduce chemical cues in asymmetric morphologic changes, according to the local gradients. For example, signaling molecules such as Netrin-1 (Toriyama et al., 2013), BDNF (Myers and Gomez, 2011), NGF (Turney et al., 2016), and semaphorins (Woo and Gomez, 2006) regulate axonal guidance by asymmetrically 
modulating the generation of mechanical force at the level of adhesion points/molecular clutches. The gradients of attractive or repulsive guidance cues have also been found to induce the asymmetrical activation of translation proteins that build up or disassemble the cytoskeleton, respectively, leading to axon steering (van Horck et al., 2004; Lin and Holt, 2007).

A crucial point to understand is how ectopic force generation could help in understanding the molecular mechanisms of axonal growth and in designing novel nerve regeneration strategies. Nanotechnology has strongly contributed to new knowledge in the field. In fact, nanotechnology-based strategies can be used to generate extremely low mechanical forces, similar to those generated endogenously, for example, during growth-cone mediated elongation, by mimicking the downstream effects of chemical cues. Using beads manipulated by optical traps, a shear stress of $0.17 \mathrm{pN}$ was generated, which was enough to turn the GC direction in response to the shear (Wu et al., 2012). Similarly, by using magnetically-manipulated nanoparticles (NPs), we previously demonstrated that a force of $0.5 \mathrm{pN}$ was enough to preferentially align PC12 neurites along the direction imposed by the mechanical force (Riggio et al., 2014). We recently used the dragging force generated with a permanent magnet to stretch the neurites of PC12 cells labeled by magnetic NPs. We found that the onaxis component of the generated force oriented from the soma to the tip induces growth, under the condition of constant loading (Raffa et al., 2018). In this article, the "action at distance" exerted by magnetic fields on NP-labeled axons was used to understand the effects of extremely low mechanical forces on hippocampal neurons.

\section{Materials and Methods}

Animals

Animal procedures were performed in strict compliance with protocols approved by Italian Ministry of Public Health and of the local Ethical Committee of University of Pisa, in conformity with the Directive 2010/ 63/EU. C57BL/6J mice (post-natal day, P0-P1) were used. They were kept in a regulated environment $\left(23 \pm 1^{\circ} \mathrm{C}, 50 \pm 5 \%\right.$ humidity) with a $12 / 12 \mathrm{~h} \mathrm{light/dark}$ cycle (lights on at 8 A.M.) with food and water ad libitum.

\section{Cell culture}

The dissection of both hippocampi of P0-P1 C57BL/6 mice of both sexes was conducted in a solution of D-glucose $6.5 \mathrm{mg} \mathrm{ml}^{-1}$ in DPBS (Invitrogen); the tissue was digested in $0.25 \%$ trypsin (Invitrogen) and $1 \%$ DNase (Sigma, DN25, $400 \mathrm{U} \mathrm{mg}^{-1}$ ), and the cells were mechanically dissociated. Cells were seeded in high-glucose DMEM with $10 \%$ fetal bovine serum, $100 \mathrm{IU} \mathrm{ml} \mathrm{m}^{-1}$ penicillin, $100 \mu \mathrm{g} \mathrm{ml}^{-1}$ streptomycin, and 2 mM Glutamax (Invitrogen) on poly-D-lysine (PDL) coated surfaces at a density of 160 cells $/ \mathrm{mm}^{2}$, unless stated otherwise. Cells were incubated at $37^{\circ} \mathrm{C}$ in a saturated humidity atmosphere containing $95 \%$ air and $5 \%$ $\mathrm{CO}_{2}$. Four hours later, the medium was replaced with Neurobasal-A medium (Invitrogen) modified with B27 (Invitrogen), 2 mм Glutamax (Invitrogen), $50 \mathrm{IU} \mathrm{ml}{ }^{-1}$ penicillin, $50 \mu \mathrm{g} \mathrm{ml}^{-1}$ streptomycin, and $5 \mu \mathrm{g}$ $\mathrm{ml}^{-1} \mathrm{NP}$ (unless stated otherwise).

NPs

Magnetic NPs have an inorganic core of iron oxide and an organic shell preventing aggregation.

Feraheme NPs (hereafter namely NP@3nm) have a core of iron oxide crystallites with diameter of $\sim 3.25 \mathrm{~nm}$ and saturation magnetization of $25.2 \mathrm{Am}^{2} \mathrm{~kg}^{-1}$. The outer layer consists of a low molecular weight, semisynthetic carbohydrate hydrodynamic, corresponding to a hydrodynamic diameter of $30 \mathrm{~nm}$ (Bullivant et al., 2013).

NP@25nm are polyethyleneimine (PEI; $25 \mathrm{kDa}$ ) coated $\mathrm{Fe}_{3} \mathrm{O}_{4} \mathrm{NPs}$, which we have extensively characterized elsewhere (Calatayud et al.,
2013). The particles have a magnetic core of $25 \pm 5 \mathrm{~nm}$, saturation magnetization $58 \mathrm{Am}^{2} \mathrm{~kg}$, and a hydrodynamic diameter of $50 \mathrm{~nm}$.

NP@75nm are magnetite NPs (Fluid-MAG-ARA, Chemicell) with a core of iron oxide $\sim 75 \pm 10 \mathrm{~nm}$ in diameter and saturation magnetization of $59 \mathrm{Am}^{2} \mathrm{~kg}^{-1}$, as stated from the supplier. The outer layer is made of glucuronic acid and the hydrodynamic diameter is $100 \mathrm{~nm}$.

\section{Magnetic field}

Experiments were conducted in $35 \mathrm{~mm}$ Petri dishes placed inside a Halbach-like cylinder magnetic applicator, which provided a constant magnetic field gradient of $46.5 \mathrm{~T} \mathrm{~m}^{-1}$ in the radial centrifugal direction (Riggio et al., 2014; Raffa et al., 2018).

\section{Stretching assay}

Cells were seeded in $35 \mathrm{~mm}$ Petri dishes (300,000 cells per dish). Alternatively, an in vitro regeneration model was generated by using the microfluidic chip (Xona, RD150) and seeding 90,000 cells in both top of the soma side. Alternatively, for confocal and electron imaging, 80,000 were seeded on a $14 \mathrm{~mm}$ glass coverslip. Particles were added $4 \mathrm{~h}$ after seeding (day in vitro, DIV0.17).

At DIV1, the culture was placed in the magnetic applicator $\left(\mathrm{M}^{+}\right.$ group) or outside ( $\mathrm{M}^{-}$group). At DIV3 (where not differently stated) samples were fixed and stained with crystal violet $0.01 \%$ or, alternatively, stained for immunofluorescence or for electron microscopy.

\section{Immunostaining}

For immunostaining, fixed samples were permeabilized with $0.5 \%$ Triton X-100 and blocked with 5\% goat serum (GS) at room temperature. Primary antibody TUBB3 (Sigma, \#T8578, 1:500) or KDEL (Thermo Fisher Scientific, PA1-013, 1:200) was diluted in 3\% GS/0.2\% Triton X-100 in PBS and incubated overnight at $4^{\circ} \mathrm{C}$. Samples were then washed and incubated with secondary antibody (Life Technologies, \#06380 or \#AB_2633280, 1:500) and Hoechst 33342 (Invitrogen, H3570, 1:1000) or Syto RNA select (Invitrogen, S32703, 1:6000). All images were acquired using a fluorescent microscope (Nikon, TE2000-U) or a laser scanning confocal microscope (Nikon, Eclipse Ti) or a Zeiss LSM 880 with Airyscan confocal laser-scanning microscope (Carl Zeiss).

\section{Particle localization}

Fluorescent NP@75nm (Chemicell, nanoscreenMAG-ARA/R) have been used. For particle quantification, the voxel number and volume were calculated (Fiji, 3D particle counter). 3D surface plot plugin has been used for visualization. Re-slice command has been used for $Z$ stack imaging.

\section{Calcium imaging}

Neurons were exposed to $2 \mu \mathrm{M}$ Fluo-4AM (Invitrogen, F14201) in cell culture medium for $30 \mathrm{~min}$ at $37^{\circ} \mathrm{C}$ and $5 \% \mathrm{CO}_{2}$, before replacing the cell medium and placing the Petri in a suitable thermostated chamber for confocal imaging by a Zeiss LSM 880 with Airyscan confocal laserscanning microscope (Carl Zeiss). Excitation wavelength was set at $488 \mathrm{~nm}(10-20 \mu \mathrm{W}$ power emission at objective), whereas emission was collected in the 500 to $560 \mathrm{~nm}$ range. A $63 \times$ Apochromat NA=1.4 oilimmersion objective was used, setting the pinhole aperture of the confocal system at 1 airy unit. Pixel dwell time was adjusted to $1.52 \mu \mathrm{s}$, and $512 \times 512$ pixel images were collected by taking four averages per line scan.

\section{Drug treatment}

From DIV1 to DIV3, cell cultures were maintained in growth medium supplemented with blebbistatin (Sigma, \#B0560) or cytochalasin-B (Sigma, \#C6762) or paclitaxel (Sigma, \#T7402) or nocodazole (Sigma, \#SML1665) or cycloheximide (Sigma, \#C7698).

\section{Toxicity test}

Toxicity of NPs and drugs was evaluated by performing dose-response assays. At DIV3, cells were fixed, stained with crystal violet $0.01 \%$, and the number of viable neurons was counted. IC25 was calculated by regression analysis. 3-(4,5-dimethylthiazole-2-yl)-2,5-diphenyl tetrazolium bromide (MTT; Sigma) cell proliferation assay was used, according 


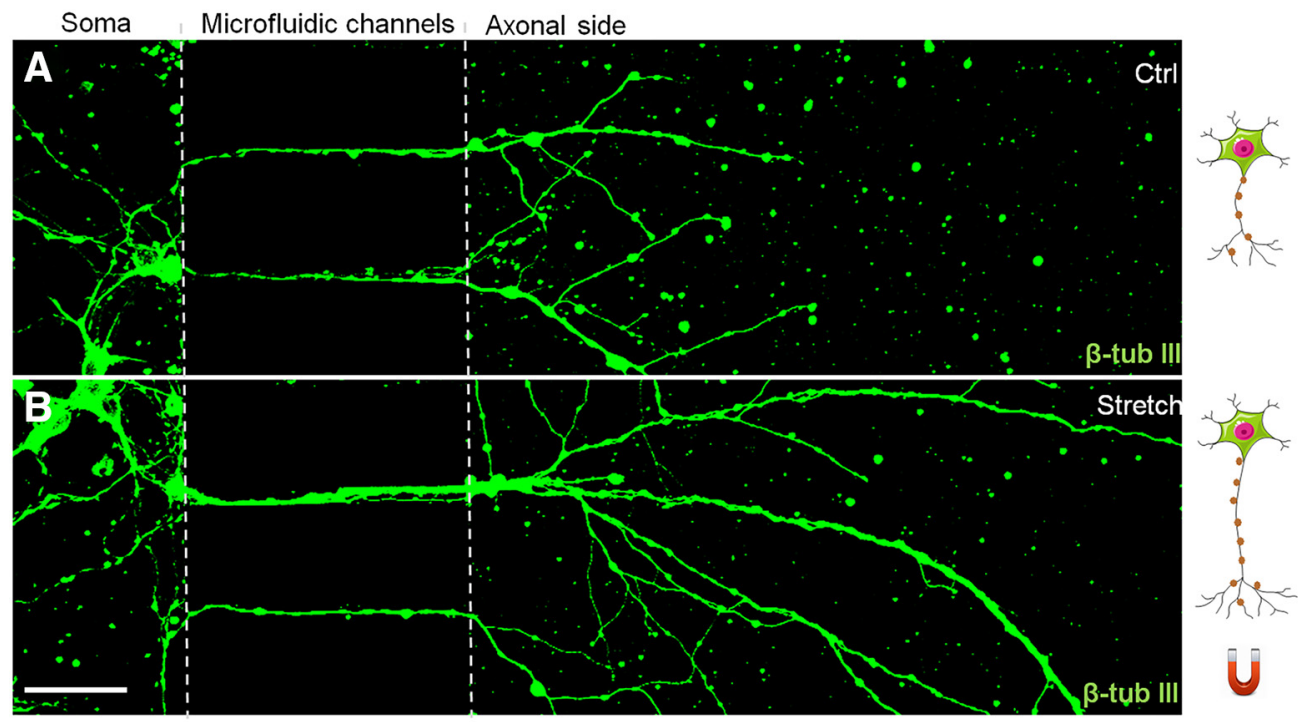

Figure 1. Tip-growth $(\boldsymbol{A})$ and stretch-growth $(\boldsymbol{B})$. Cells were cultured in the microfluidic device. The dotted lines outline the soma compartment (left), the chamber with microfluidic channels (middle), and the axonal compartment (right). Only axons can cross the channels. NPs@75nm added at DIV0.17 and anti- $\beta$-tubulin III staining performed at DIV4. Scale bar: $50 \mu \mathrm{m}$.

to manufacturer instructions. The absorbance was measured on a Versamax microplate reader (Molecular Devices) at a wavelength of $570 \mathrm{~nm}$ with the background subtracted at $690 \mathrm{~nm}$.

An iron assay kit (DIFE-250, QuantiChrom) was performed on cell lysate for intracellular iron quantification, and the absorbance was measured at a wavelength of $590 \mathrm{~nm}$.

\section{Image analysis}

The analysis of the elongation and sprouting was performed by using image analysis software ImageJ. Neurite length $l$ (primary, secondary, and tertiary processes) was evaluated by using the plugin NeuronJ (Popko et al., 2009), and 90 non-interconnected primary axons were analyzed from $10 \times$ magnification images (randomly acquired).

For axon thickness, a population of 30 axons was analyzed from $10 \times$ magnification images (randomly acquired). For each axon, the longest path $l$ has been considered, and the thickness $s$ was calculated as $s=A / l$, $A$ being the axon area related to that path. Area was calculated from images after threshold normalization, binary conversion.

For the branching patterns identification, a population of 30 neurons was analyzed from $40 \times$ magnification images (randomly acquired) by using the semi-automated program Bonfire (Kutzing et al., 2010).

Transmission electron micriscopy (TEM) analysis

Samples were fixed with $1.5 \%$ glutaraldehyde in $0.1 \mathrm{~m}$ sodium cacodylate buffer, $\mathrm{pH}$ 7.4.

After rinses with the same buffer, cells were postfixed in reduced osmium solution $\left(1 \% \mathrm{OsO}_{4}, 1 \% \mathrm{~K}_{3} \mathrm{Fe}(\mathrm{CN})_{6}\right.$, and $0.1 \mathrm{~m}$ sodium cacodylate buffer), stained with our homemade staining solution (Convertino et al., 2013), dehydrated in a growing series of ethanol, and flat-embedded in Epoxy resin.

Ultra-thin sections ( $90 \mathrm{~nm}$ ) were cut with a UC7 LEICA ultramicrotome (UC7, Leica Microsystems) and collected on 300 mesh copper grids (Electron Microscope Science).

Images for morphologic characterization have been collected with a transmission electron microscope Zeiss LIBRA 120 Plus operating at 120 $\mathrm{KeV}$ equipped with an in-column $\omega$ filter.

\section{Patch-clamp recordings}

Primary hippocampal cultures were recorded as previously described (Siano et al., 2019). Under continuous perfusion with Tyrode's solution containing the following: $150 \mathrm{~mm} \mathrm{NaCl}, 4 \mathrm{~mm} \mathrm{KCl}, 1 \mathrm{~mm} \mathrm{MgCl}_{2}, 4 \mathrm{~mm}$ $\mathrm{CaCl}_{2}, 10 \mathrm{~mm}$ glucose, and 10 mm HEPES; pH 7.4 with $\mathrm{NaOH}$, cells were whole-cell patched with borosilicate glass pipettes (pulled to a resistance of 5-7 M $\Omega$ using a P-97 Sutter puller) filled with an internal solution containing the following: $145 \mathrm{~mm} \mathrm{~K}$-gluconate, $2 \mathrm{mM} \mathrm{MgCl}_{2}, 10 \mathrm{~mm}$

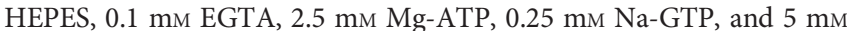
phosphocreatine; pH 7.35 with KOH. Spontaneous EPSCs (sEPSCs) were recorded in voltage-clamp configuration while holding the neuron at $-70 \mathrm{mV}$. The resting membrane potential was measured for at least $1 \mathrm{~min}$ in $\mathrm{I}=0$ configuration. Spiking activity was quantified in current clamp configuration, by adjusting the holding current to obtain an initial membrane potential of $-60 \mathrm{mV}$ before delivering a 200-pA current step. Data were acquired using a MultiClamp 700A amplifier, connected to a Digidata 1322A digitizer (Molecular Devices) and analyzed using Clampfit 10.7 (Molecular Devices). sEPSCs were analyzed using the template-based event detection function of Clampfit, as previously described (Testa et al., 2019).

\section{Statistical analyses}

Data were plotted and analyzed with GraphPad software, version 6.0. Significance was set at $p \leq 0.05$. Statistical power analyses have been performed with G-power software.

\section{Results}

\section{The stretching protocol}

Recently, we demonstrated in PC12 cell line that neurite labeling with iron oxide magnetic NPs enables them to be stretched through the dragging force generated by an external permanent magnet (Raffa et al., 2018). In this article, magnetic labeling was used to stretch the axons of hippocampal neurons isolated from P1 mouse pups. The labeling and stretching protocols consisted in incubating neurons with cell culture medium modified with NPs $5 \mu \mathrm{g} \mathrm{ml}^{-1} 4 \mathrm{~h}$ after plating and the application of a magnetic field $\left(\nabla \vec{B}=40 \mathrm{Tm}^{-1}\right.$, stretch group) or a null magnetic field (Ctrl group) at DIV1, respectively. Figure 1 shows the neurons grown in microfluidic devices to separate the somatodendritic compartment from the axons. A comparison between the tip-growth (control; Fig. $1 A$ ) and stretch-growth (Fig. $1 B$ ) indicates the dramatic effect of the stretch on the length of axons after $3 \mathrm{~d}$ of stretching.

We tested various iron oxide NPs with a similar saturation magnetization $M_{s}$, but different iron oxide core sizes (@3nm, @25nm, @75nm). The length of outgrowth was measured. NPs were diluted in cell media at the non-toxic concentration of $5 \mu \mathrm{m}$ $\mathrm{ml}^{-1}$, and the stretching time was $48 \mathrm{~h}$ (Fig. 2C). All particles induced elongation: the length increase for stretched axons with respect to the non-stretched condition was $11.4 \pm 3.3 \%, 75.8 \pm$ 
A

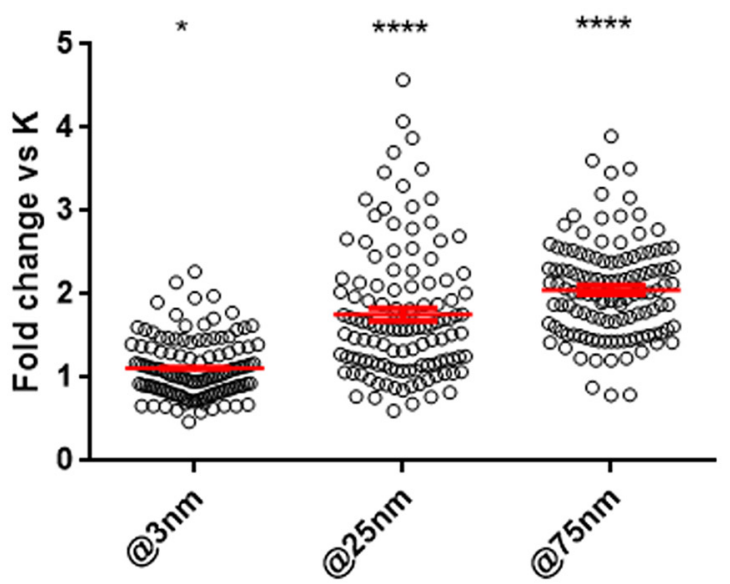

C

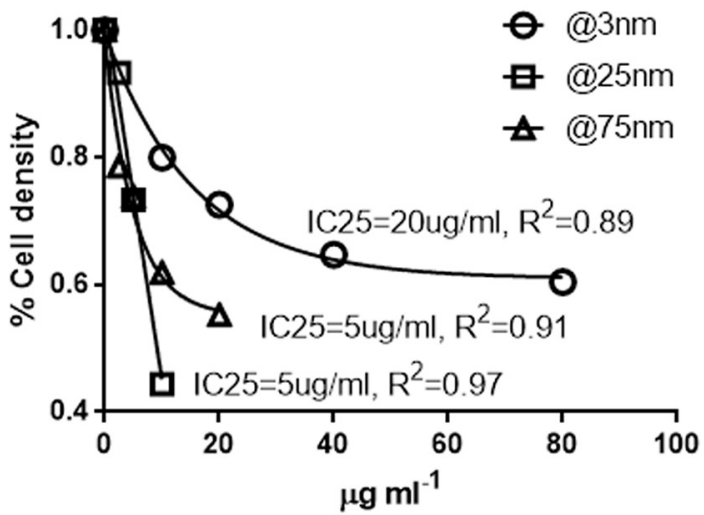

B

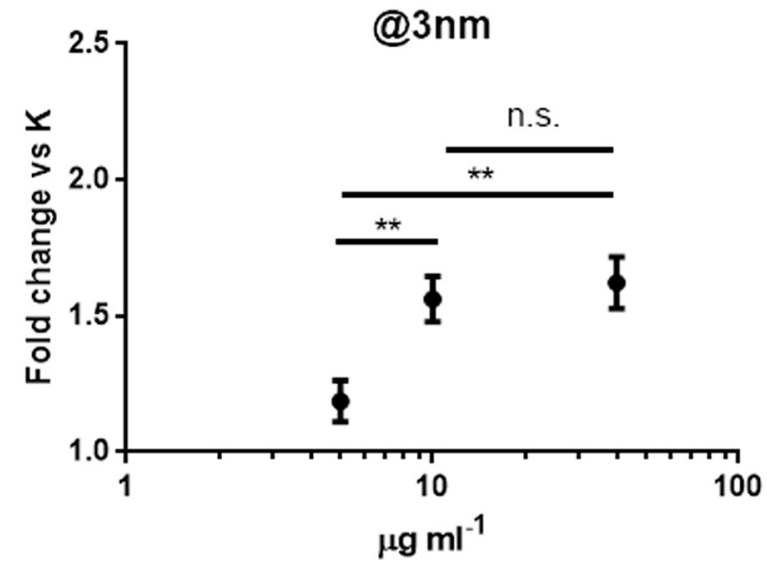

E

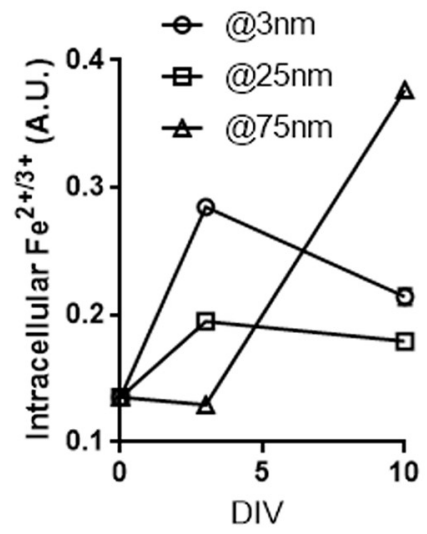

Figure 2. Stretch promotes axonal elongation. A, Stretch assay with magnetic beads @3nm, @25nm, and @ $975 \mathrm{~nm}$. Fold change of axon length in stretched versus non-stretched conditions (scatter dot plot, mean $\pm \mathrm{SEM}$ ). NP concentration $5 \mu \mathrm{g} \mathrm{ml}^{-1} ; n=120$ neurons from four independent assays; $t$ test for unpaired data two-tailed (each dataset versus its own control group, i.e., un-stretched group); $p=0.0129, t=2.505, \mathrm{df}=2.38$ for $@ 3 \mathrm{~nm} ; p<0.001, t=9.681, \mathrm{df}=238$ for $@ 25 \mathrm{~nm} ; p<0.0001, t=17.45$, df $=238$ for @75nm. $B$, Stretch growth increases with an increase in the concentration of the magnetic beads in cell growth medium. Fold change of axon length in stretched versus non-stretched conditions (mean \pm SEM), NP@3nm; $n=30$, one-way ANOVA with post hoc Tukey's HSD test, $p=0.0009, F=7.632$. C, Dose-response assay: the number of neurons was counted at DIV3; $n=4$, one-phase decay regression analysis. $R^{2}=$ $0.89,0.91,0.97$ for @3nm, @25nm, @75nm, respectively. IC25 = 5 $\mu \mathrm{g} \mathrm{ml}^{-1}, 5 \mu \mathrm{g} \mathrm{ml}^{-1}, 20 \mu \mathrm{g} \mathrm{m}^{-1}$ for @3nm, @25nm, @75nm, respectively. D, Intracellular levels of Fe ${ }^{2+13+}$ versus time (NPs added at DIV0.17). $E$, MTT assay of NP@75nm. Dose-response viability assay (box plot, min-to-max); $n=8$. One-way ANOVA, $p=0.25, F=1.461$. Statistical analyses: ${ }^{*} p<0.05$, ${ }^{* *} p<$ $0.01,{ }^{* * *} p<0.0001$, n.s., not statistically significant.

6.4\%, and 105.4 $\pm 7.2 \%$ for @3nm, @25nm, and @75nm, respectively (Fig. 2A). Interestingly, even when the smallest particles (@3nm) were incubated at higher concentrations, they were able to induce sustained neurite elongation (Fig. $2 B$ ). These observations suggest that NP-mediated stretch-growth is a general mechanism (i.e., not particle-specific). On the other hand, NP@75nm had the most pronounced effect in terms of axonal lengthening (Fig. 2A). Additionally, NP@75nm seem to be the most stable intracellularly, as the onset of inorganic core degradation, documented by the increase in the levels of intracellular iron, appears after DIV3 (Fig. 2D). Thus, we chose to perform subsequent experiments with @75nm particles.

The following formula was used to estimate the local force generated in the axon:

$$
\mathrm{F}=\rho V M_{s} \nabla \vec{B}
$$

where $\rho$ is the density of iron oxide, $V$ is the total volume occupied by the iron core of the NPs in the neurite, and $B$ is the magnetic field.

\section{NPs stimulate stretch-growth}

Fluorescently labeled NPs@75nm were used to detect the localization of these particles in neurons. Fluorescence was easily detected in the cytoplasm of both cell soma and neurites (Fig. $3 A) . Z$ stack reconstruction showed NPs as small puncta in the cytoplasm (Fig. $3 A$, inset). The analysis of the neuron volume reconstruction (Fig. $3 B 1$ ) via $Z$ stack re-slice showed the absence of particles in the nucleus (Fig. 3B2) but uniform particle distribution in the axon (Fig. 3B3). This was a similar result to previous observations that we collected in PC12 cells (Raffa et al., 2018). Particle localization in the axon was also confirmed by TEM analysis, and NPs were found not only to stick at the axonal cell membrane (Fig. 3C, white arrows) but also to localize within the axoplasm (Fig. 3C, black arrows). Therefore, NP magnetic labeling is a protocol for stretching the entire axon tract, and force generation is not limited to the GC but involves the whole axon.

The volume of NPs localized in the axon was estimated by calculating the volume occupied by red fluorescent voxels in the region of interest (ROI) corresponding to the volume of the axon 

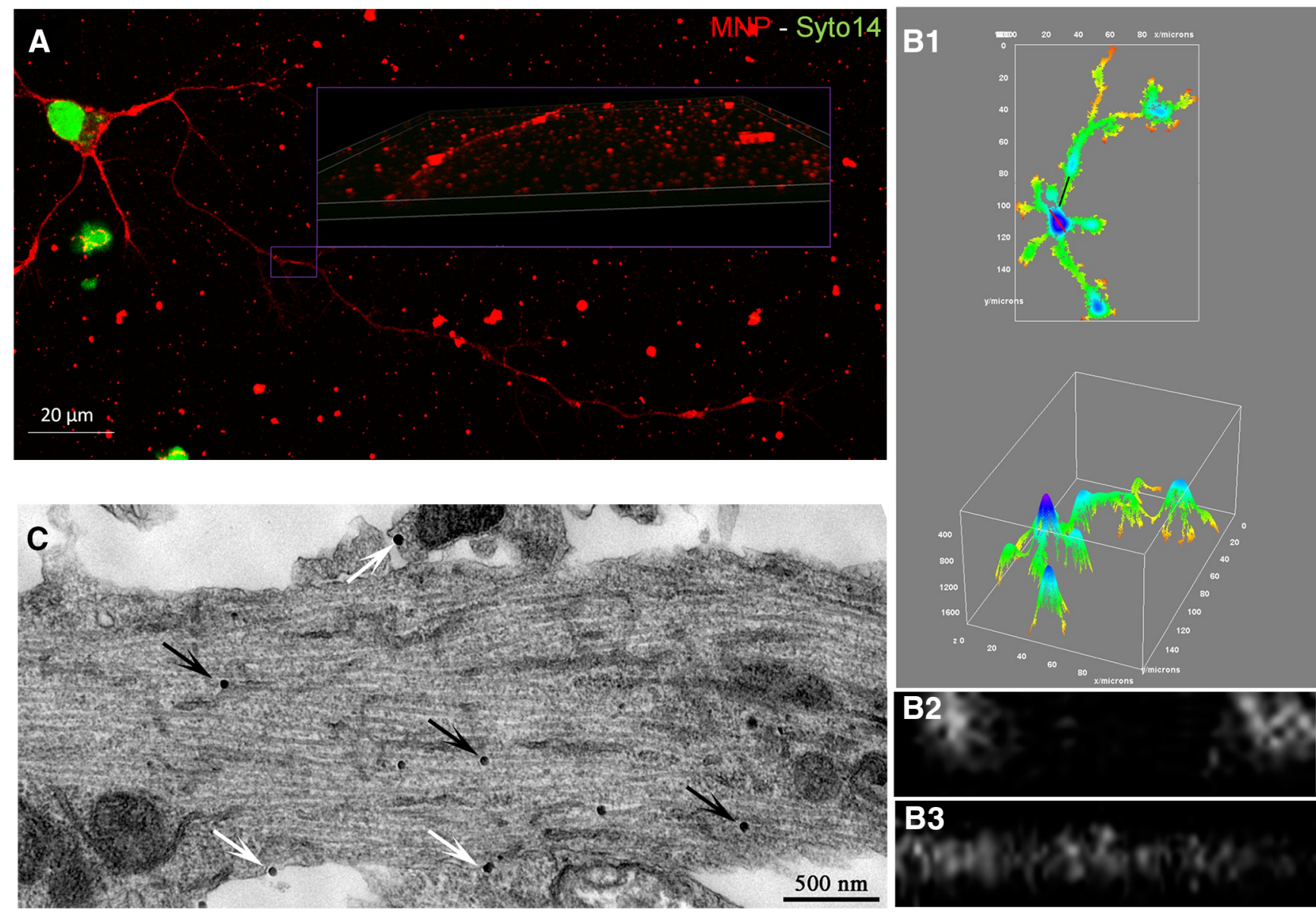

Figure 3. Imaging of cells incubated for $72 \mathrm{~h}$ with fluorescent NP@75nm. A, Confocal imaging. NPs localized in neuronal cytoplasm included the axoplasm where small puncta appear (inset: $Z$ stack reconstruction). B1, 3D surface plot, top view, and 3D view. Re-slice of cell soma (red line) shows no particles in the nucleus (B2). Re-slice of axon (black line) shows a uniform distribution of fluorescent puncta (B3). C, TEM analysis. Representative micrograph of a stretched axon. Black and white arrows highlight NPs in the axoplasm and NPs at the membrane, respectively.

selected from the bright field image. The volume of particles was found to be $1.65 \pm 0.42 \mu \mathrm{m}^{3}$ for the control group (corresponding to $3.48 \pm 0.88 \mathrm{pg}$ of iron oxide, $n=6)$ and $3.05 \pm 0.29 \mu \mathrm{m}^{3}$ for the stretch condition (corresponding to $6.43 \pm 0.61 \mathrm{pg}$ of iron oxide, $n=6$ ). This difference between the two conditions reflects the change in length (stretched axons increased 2.05 times in length and 1.85 times in particle number). We also performed a direct counting of NPs from TEM images, and we measured the number of NPs per unit of neurite volume $\left(1.16 \pm 0.31 \mathrm{NP} / \mu \mathrm{m}^{3}\right.$, $n=20$ ). By modeling the axon as a cylinder (diameter and length given by the mean of data distribution shown in Fig. $4 A 1, B$, respectively), we estimated that the volume of particles in stretched axons was $3.72 \pm 0.99 \mu \mathrm{m}^{3}$ (corresponding to $7.84 \pm$ $2.09 \mathrm{pg}$ of iron oxide), which is in good agreement with confocal measurements. By substituting these values into Equation 1, the mean force generated on-axis was estimated to be $7.59 \pm 0.72$ $\mathrm{pN}$ (using confocal data) or $9.25 \pm 2.47 \mathrm{pN}$ (using TEM data). This extremely low force was found to double the axon length after $48 \mathrm{~h}$ of continuous stretching (Fig. 4A1). A strong increase in length was also associated with secondary (Fig. 4A2) and tertiary (Fig. 4A4) axonal processes in stretched versus non-stretched conditions. Interestingly, force also increased the number of branching points (Fig. 4A3,A5). The observed elongation was growth, as highlighted by the axon caliber measurement (not statistically significant difference between stretched and nonstretched conditions; Fig. 4B). In addition, neither the magnetic field $\left(\mathrm{NP}^{-} \mathrm{M}^{-}\right)$nor the particles $\left(\mathrm{NP}^{+} \mathrm{M}^{-}\right)$alone affected axonal lengthening (mean \pm SEM was $129.9 \pm 6.34,113.1 \pm 8.46$, and $132 \pm 7.13 \mu \mathrm{m}$ for $\mathrm{NP}^{-} \mathrm{M}^{-}, \mathrm{NP}^{-} \mathrm{M}^{+}$, and $\mathrm{NP}^{+} \mathrm{M}^{-}$, respectively; one-way ANOVA, $p=0.20, F=1.64$ ).

Using our previous model (Raffa et al., 2018), we estimated an elongation rate of $1.95 \pm 0.06 \mu \mathrm{m} \mathrm{h}^{-1}$ in spontaneous elongation and $6.00 \pm 0.15 \mu \mathrm{m} \mathrm{h}^{-1}$ in stretch-growth (corresponding to $0.66 \pm 0.02 \mu \mathrm{m} \mathrm{h}^{-1} \mathrm{pN}^{-1}$, similarly to the elongation rate previously identified; Zheng et al., 1991). In order to experimentally validate the increase in the elongation rate in stretched versus non-stretched conditions, we performed intracellular calcium imaging with Fluo-4AM. Experimental data revealed a strong attenuation of the signal in the condition of stretch-growth compared with spontaneous elongation, in line with the evidence that high axonal elongation rates are associated with low $\mathrm{Ca}^{2+}$ transient, whereas low axonal elongation rates are associated with high $\mathrm{Ca}^{2+}$ transient (Rosenberg and Spitzer, 2011; Fig. $5 A 3)$. Interestingly, within $30 \mathrm{~min}$ of the magnet removal (Fig. $5 B$ ), fluorescence intensity reached control values. This is in line with the hypothesis that a constant load is required for stretchgrowth (Raffa et al., 2018).

To assess whether stretch-growth could affect cell maturation, primary hippocampal neurons were probed with patch-clamp electrophysiological recordings. Stretched neurons showed, at DIV7, a significant increase in the frequency (i.e., shorter interevent intervals), but not in the amplitude, of sEPSCs (Fig. 6). At 


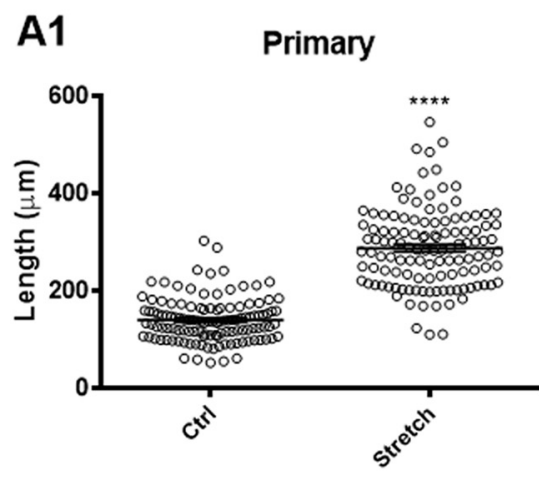

A4

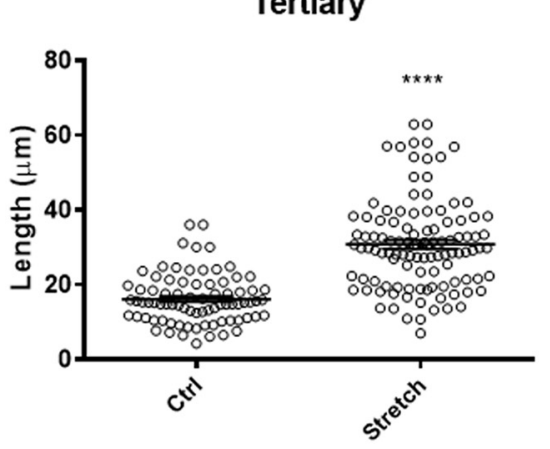

A2

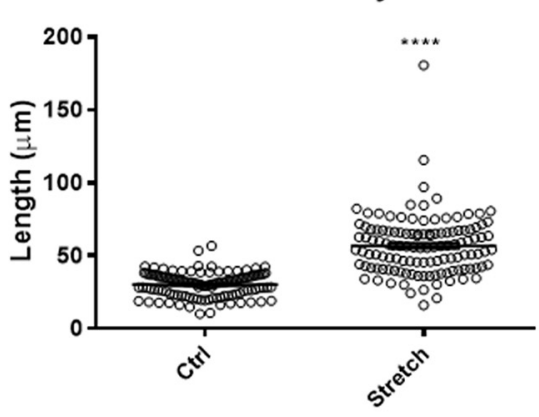

A5

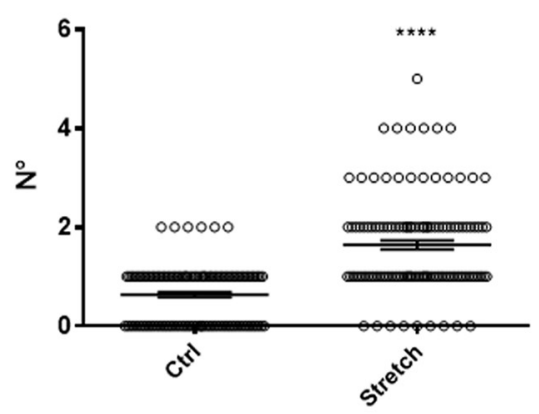

A3

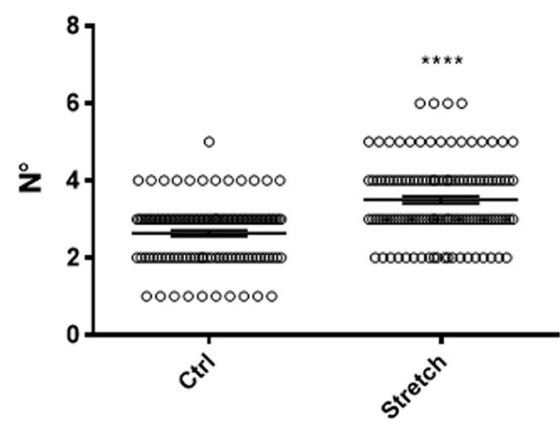

B

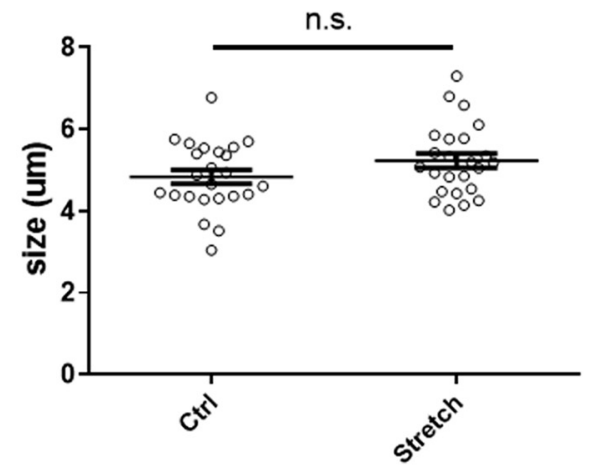

Figure 4. Stretch growth assay, $\mathrm{NP} @ 75 \mathrm{~nm} 5 \mu \mathrm{g} \mathrm{ml}^{-1}$. A1, Length of primary axons, $p<0.0001, t=17.45, \mathrm{df}=238$. A2, Length of secondary processes, $p<0.0001, t=13.2, \mathrm{df}=238$. $\mathbf{A}$, Number of secondary processes, $p<0.0001, t=7.289, \mathrm{df}=238$. A4, Length of tertiary processes, $p<0.0001, t=9.694, \mathrm{df}=186$. A5, Number of tertiary processes, $p<0.0001, t=9.603$, $\mathrm{df}=238 . B$. Axonal caliber, $p=0.12, t=1.597, \mathrm{df}=46$. For all panels: scatter dot plot (mean $\pm \mathrm{SEM}$ ); $t$ test for unpaired data, two-tailed, $n=120$ neurons from four independent assays. Statistical analyses: ${ }^{* * *} p<0.0001$, n.s., not statistically significant.
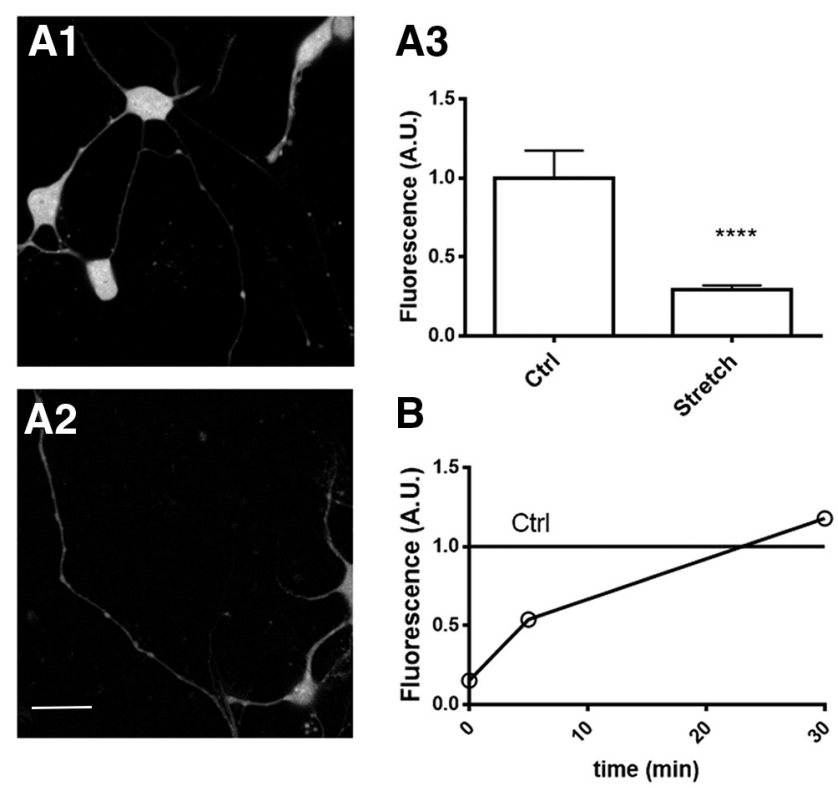

Figure 5. Calcium imaging. Fluo-4AM fluorescence (mean \pm SEM) from non-stretched neurons (A1) and stretched neurons imaged within 5 min after magnet removal (A2) and data analysis $(\boldsymbol{A} 3 ; n=18$ neurons from three independent replicates, unpaired $t$ test twotailed, $p=0.0003, t=4063, \mathrm{df}=34$ ). Stretched axons gain fluorescence levels similar to control within $30 \mathrm{~min}$ after magnet removal $(\boldsymbol{B})$. Scale bar: $20 \mu \mathrm{m}$. Statistical analyses: **** $p<0.0001$
DIV14, both the frequency and amplitude of sEPCSs were significantly higher than control cultures (Fig. 6). Stretch-growth also increased the number of action potentials discharged in response to a 200-pA current step at both DIV7 and DIV14 (Fig. 6). On the other hand, stretched and control neurons did not show significantly different resting membrane potentials (DIV7, control, $n=14, \quad-45.367 \pm 2.143 \mathrm{mV}$ vs stretch-growth, $n=14$, $-41.413 \pm 2.341 \mathrm{mV}$; Student's $t$ test, $p=0.226$; DIV14, control, $n=10,-55.991 \pm 2.108 \mathrm{mV}$ vs stretch-growth, $n=13,-56.483$ $\pm 2.179 \mathrm{mV}$; Student's $t$ test, $p=0.875$ ).

Stretch-growth shows typical hallmarks of sustained growth As the addition of new mass is required to sustain axon outgrowth, we tested the effects of the downregulation of global translation. Cycloheximide, an inhibitor of protein synthesis, was found to differentially impair tip-growth and stretch-growth, which is in line with the hypothesis that rapidly-growing axons require sustained protein translation (Fig. 7A). However, rapid axon growth also requires the rapid manufacture and supply of membrane and lipids. TEM imaging shows that stretched axons exhibit the accumulation of cisternae with an endoplasmic reticulum (ER) that extends along the axon (Fig. 8, white stars). Moreover, we observed that this effect is clearly evident both in the proximal and in the distal portion of axon. This accumulation was also confirmed by staining cells against KDEL (which is a known ER marker), and the axonal compartment showed a considerable increase in the density of the immunofluorescence signal in stretched versus non-stretched conditions (58\% 
A
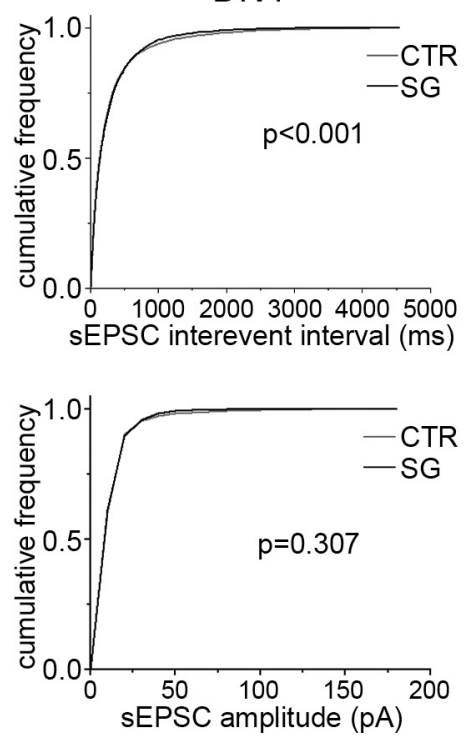

DIV7

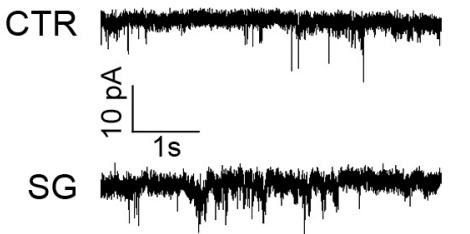

DIV14
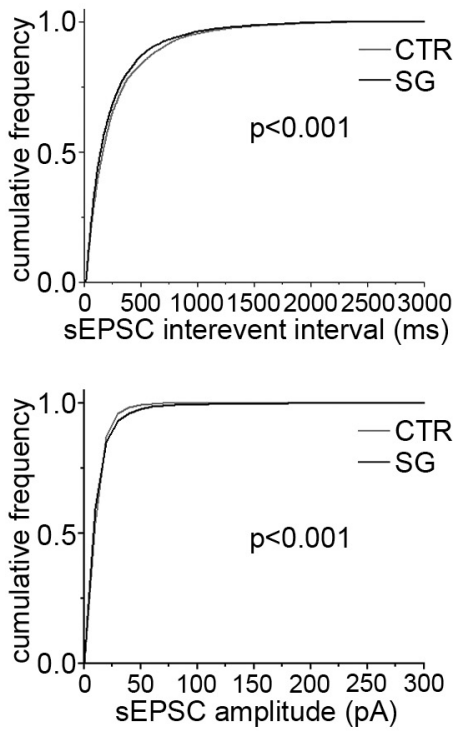

DIV14

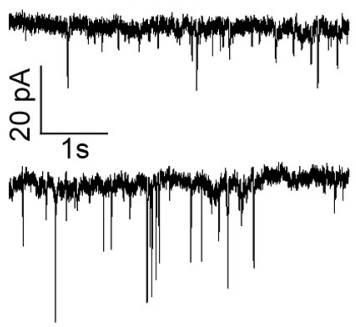

\section{B}

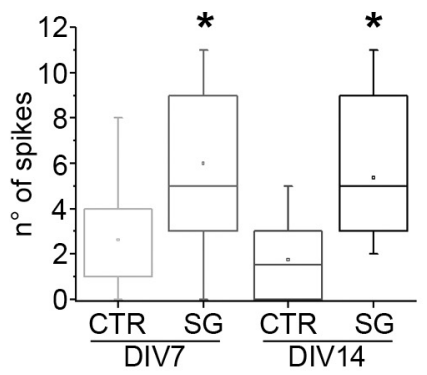

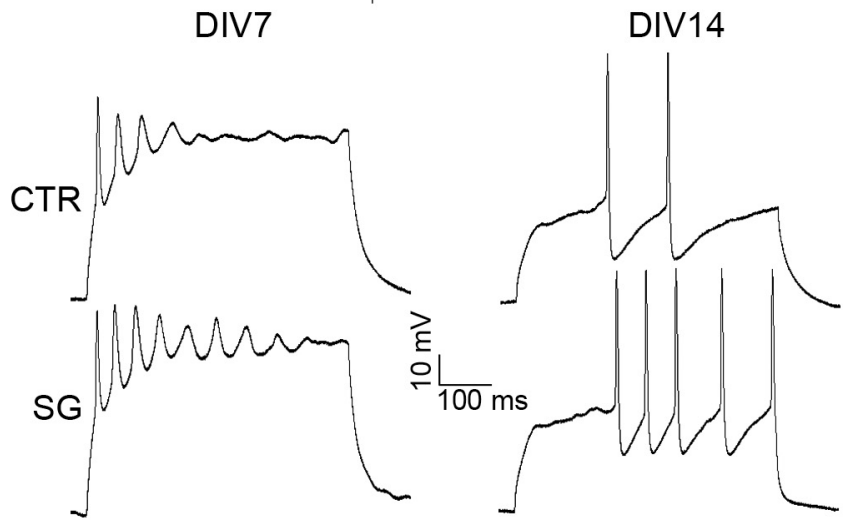

Figure 6. Patch clamp recordings on primary hippocampal neurons subjected to stretch-growth. $\boldsymbol{A}$, Cumulative frequency distributions showing at DIV7, a significant increase in the frequency, but not in the amplitude of sEPSCS in stretch-grown (SG) neurons compared with controls (CTR; two-sample Kolmogorov-Smirnov test; CTR, $n=15$; SG, $n=8$; left) and at DIV14, a significant increase in both frequency and amplitude of sEPSCS in SG versus (TR neurons (two-sample Kolmogorov-Smirnov test; (TR, $n=7$; SG, $n=10$; right). Bottom panels show representative voltage-clamp recordings from the four experimental groups. B, SG neurons fire a higher number of spikes in response to a 200-pA current injection than CTR neurons (DIV7, Mann-Whitney rank-sum test, $p=0.044$, (TR, $n=13$; SG, $n=13$; DIV14, Mann-Whitney rank-sum test, $p=0.034$, (TR, $n=8 ;$ SG, $n=11$; left) and representative current clamp recordings from the four experimental groups (right). Statistical analyses: ${ }^{*} p<0.05$.

increase, $p<0.0001$; Fig. $8 C$ ). This is in line with the view that axon outgrowth critically depends on the supply of proteins and lipids through the secretory route (Valenzuela and Perez, 2015).

In order to assess whether these rapidly-growing stretched axons still respond to neurotrophin stimulation, we tested BDNF, whose effect on hippocampal neurons has already been characterized (Labelle and Leclerc, 2000). In tip growth, we found a dose-response effect to BDNF on axon outgrowth; however, in stretch-growth, axons were found not to respond to BDNF (Fig. 9A), suggesting an interference between the two pathways or a saturating effect.
Stretch-growth induces an increase in microtubule (MT) density

Transmission electron microscopy revealed that, in stretched axons, MTs appear normal both in architecture and in polarity. However, their density seems extremely higher, resulting in a strong reduction of free axonal cytoplasm and in cytoskeletal bundles accumulation (Fig. 8A, stretch group). To avoid the possibility this effect is related to the distance from the neuron soma, we analyze axon both in the proximal (closed to GC) and in the distal tract. In the stretched axons, MT linear density is higher than in control axons $(9.9 \pm 1.5$ and $7.2 \pm 1.2 \mathrm{MTs} / \mu \mathrm{m}$, 
respectively, $n \geq 25, \quad t$ test, $p<$ 0.0001 ; Fig. $8 B$ ), resulting in a reduction of the inter-distances between MTs. It is thus possible that force promotes MT polymerization along the axon shaft, with unknown mechanisms. This also supports the idea of a more general mechanism of addition of axonal MT mass, which is generated in the shaft, rather than in GCs, and shifts anterogradely toward the tip (Roossien et al., 2013; Voelzmann et al., 2016).

In order to understand the effect of stretch-growth on MT dynamics, we tested neurons by pharmacological treatment with nocodazole or paclitaxel, which induce MT destabilization and stabilization, respectively. Treatment with nocodazole, which interferes with the polymerization of MTs, had no effect on tipgrowth, but led to a total inhibition of stretch-growth when administered at the concentration of $1.8 \mathrm{ng} \mathrm{ml}^{-1}$ (Fig. 9A). However, paclitaxel, which stabilizes MTs by binding to the $\mathrm{N}$ terminus of $\beta$-tubulin, had no effect on tip-growth and stretch-growth, when administered at the concentration of $0.025 \mathrm{\mu g} \mathrm{ml}^{-1}$ (Fig. $9 E)$. By increasing the paclitaxel concentration $\left(0.05 \mu \mathrm{g} \mathrm{ml}^{-1}\right)$, we found a total inhibition of both tip-growth and stretchgrowth (Fig. $7 B$ ).

In conclusion, we found that nocodazole, but not paclitaxel, differentially influenced stretch-growth versus tip-growth.

We also tested the effect of stretch-growth after perturbation of actin filaments. By inhibiting the activity of myosin-II with blebbistantin (B) (used at the non-toxic concentration of $0.4 \mu \mathrm{g}$ $\mathrm{ml}^{-1}$ ), we found an increase in the number of branch points both in spontaneous elongation and stretch-growth (Fig. 9D). These results were consistent with the reported activity of myosin-II as a negative regulator of minor process extension (Kollins et al., 2009). The effect was additive: the increase in axonal branching was very similar in the conditions $\mathrm{B}^{-}(1.68$-fold change) versus $\mathrm{B}^{+}$(1.73-fold change) and in the conditions $\mathrm{M}^{-}$ (2.23-fold change) versus $\mathrm{M}^{+}$(2.30-fold change). Therefore, blebbistantin equally enhanced branching in tip-growth and stretch-growth, and there was no differential effect between the two conditions. Similarly, treatment with cytochalasin-B (Fig. $7 A$ ), which prevents actin polymerization, was found to totally inhibit both spontaneous elongation and stretch-growth, when administered at the non-toxic concentration of $0.05 \mu \mathrm{g} \mathrm{ml} \mathrm{m}^{-1}$ (Fig. 9E). By decreasing the concentration $\left(0.025 \mu \mathrm{g} \mathrm{ml}^{-1}\right.$ or below), no effects were found and tip-growth and stretch-growth conditions were identical to the corresponding no-drug conditions (Fig. 9D).

In conclusion, the inhibitors of actin dynamics, blebbistatin and cytochalasin-B, did not differentially modulate stretchgrowth versus tip-growth. Overall, this differential effect of nocodazole versus paclitaxel, blebbistatin, and cytochalasin-B indicates that enhanced MT polymerization is necessary to support stretch-growth.

\section{Discussion}

Axonal growth is motion, and motion is driven by force (Franze, 2013). Several attempts have been made to measure local forces in axons, and these studies have greatly contributed to a better
Stretch

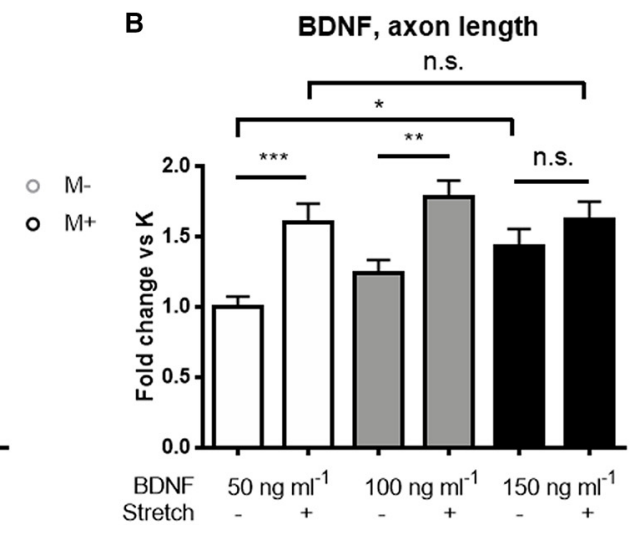

1

Figure 7. Effects of biochemical enhancement/inhibition of axonal elongation. $A, 30 \mathrm{ng} \mathrm{ml}^{-1}$ cycloheximide was added at DIV1. Tukey's HSD test. Row factor $\left(\mathrm{CH}^{-}\right.$vs $\left.\mathrm{CH}^{+}\right): p<0.0001, F=275.1$. Columns factor $\left(\mathrm{M}^{-}\right.$vs $\left.\mathrm{M}^{+}\right): p<0.0001, F=299.8 . B, B D N F$ was added at DIV1. Fold change of axon length at DIV3 in stretched versus non-stretched conditions (mean \pm SEM); $n=30$ neu$p=0.0855, F=2.495$. Interaction factor: $p=0.143, F=1.969$. Statistical analyses: ${ }^{*} p<0.05,{ }^{* *} p<0.01,{ }^{* *} p<0.001{ }^{* * * *} p$ t.

understanding of the biomechanics of axonal outgrowth. Neurons generate higher contractile forces in the $\mathrm{GC}\left(F_{c}^{G C}\right)$ via clutch engagement, and weaker contractile forces along the axon $\left(F_{c}^{A}\right)$ via the axonal actin cortex and non-muscle myosin-II (NMII)-driven contraction (Mutalik et al., 2018). Axonal contractile forces are countered by the pushing forces $\left(F_{M T}^{A}\right)$ associated with MT assembly or generated by dynein (Roossien et al., 2014). Axonal elongation occurs when the contractile force in the GC is higher than the net contractile force in the axonal shaft (assuming a similar viscosity at the axon and at the rear of the GC; O'Toole et al., 2015), i.e.,

$$
F_{c}^{G C}>F_{c}^{A}-F_{M T}^{A} .
$$

An implication of the possibility that axonal elongation pairs with this force gradient is that axon growth could result from high levels of traction force at the GC $\left(F_{c}^{G C}\right)$ or low levels of traction force $\left(F_{c}^{A}\right)$ at the axon, or high level of forces associated with MT $\left(F_{M T}^{A}\right)$. For example, in chick sensory neurons, O'Toole and colleagues found that the GC generates $2 \mathrm{nN}$ contractile force, that the axon generates $0.6 \mathrm{nN}$ of net contractile force, and that the average net force for the neuron as a whole was $1.3 \mathrm{nN}$ (O'Toole et al., 2015). As a result of this force gradient, the GC pulls the adjacent axon forward, causing a bulk flow of MTs during axonal elongation through multiple interactions (Roossien et al., 2014). Earlier work using TEM in hippocampal neurons of vertebrates helped in understanding the link between MT length, density, sliding and new mass addition during elongation. Stage 3 neurons (DIV1-DIV2) are characterized by a large increase in the number of MTs (Yu and Baas, 1994). In contrast, the transition into stage 4 (DIV3) is associated with an increase in MT length, along with a drop in MT density due to sliding. MT sliding is crucial in neurite initiation (stage 1-2 neurons) and entails the coupling of kinesis- 1 to anti-parallel MTs which slide because of the motor protein moving toward the plus end of one MT ( $\mathrm{Lu}$ et al., 2013; Oelz et al., 2018).

The contribution of MT sliding to axonal elongation is less clear because axonal MTs are prevalently parallel (80\%). However, experimental evidence supports the view that MT sliding supplies enough MT mass to sustain axonal extension (Athamneh et al., 2017; Rao et al., 2017). 

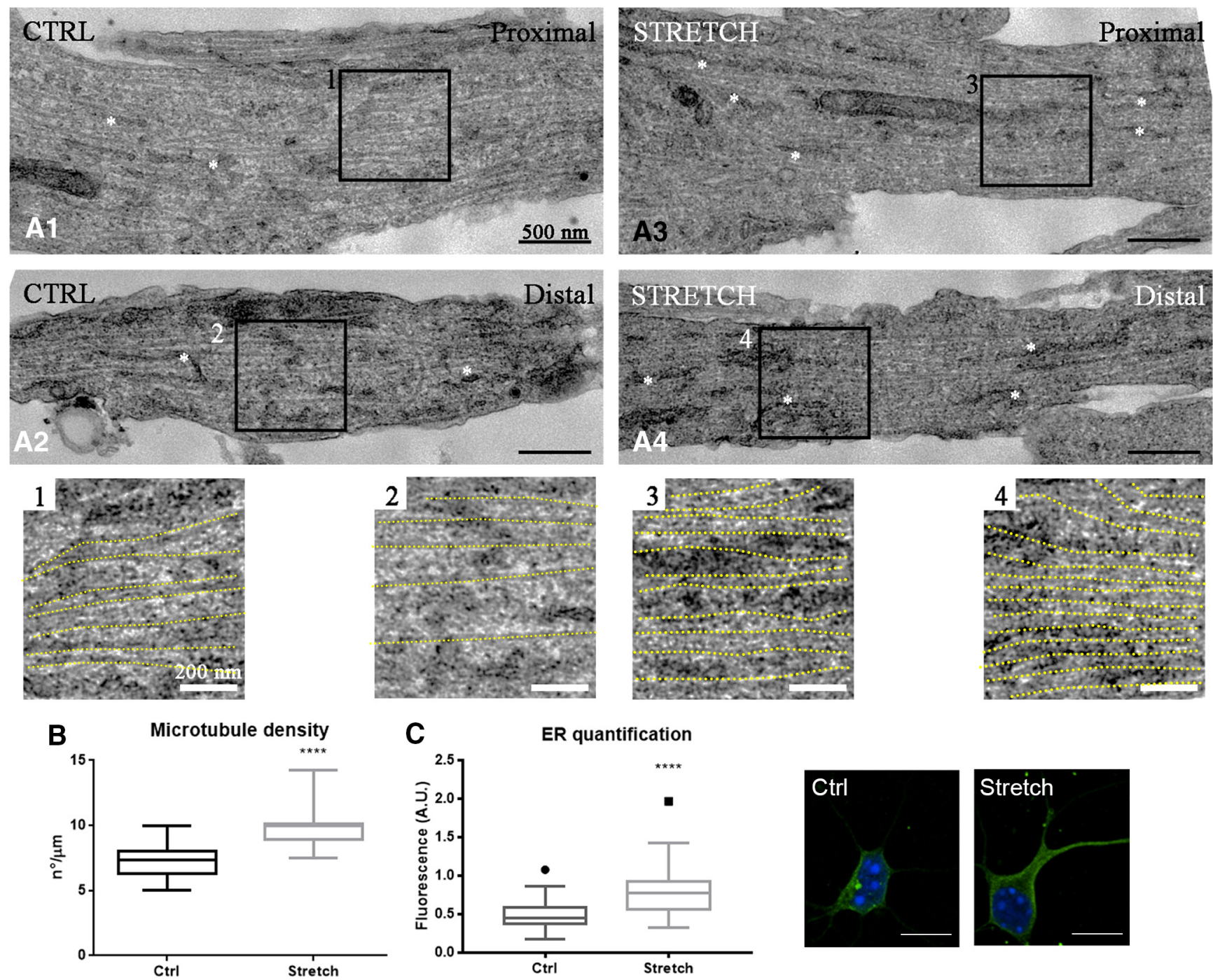

Figure 8. TEM analysis of stretched and control axons. $\boldsymbol{A}$, Representative micrographs of axonal cytoskeleton in distal and proximal axon tracts of control and stretched hippocampal neurons. White stars highlight examples of ER cysternae. Dashed yellow lines indicate MTs. $\boldsymbol{B}$, Quantification of MT linear density (box plot, min-to-max). Unpaired $t$ test, two-tailed, $p<0.0001$, $t=7.801, \mathrm{df}=61$. C, Quantification of ER (box plot, Tukey's test). KDEL fluorescence in the axonal compartment under stretched versus non-stretched conditions ( $t$ test for unpaired data, twotailed, $n=40$ neurons from four independent assays); $p<0.0001, t=4.988, \mathrm{df}=78$. Scale bar: $10 \mu \mathrm{m}$. Statistical analyses: ${ }^{*} p<0.05,{ }^{* * * *} p<0.0001$.

Our data partially differ from the current understanding of tip-growth. We observed that the stretched axons of DIV3 hippocampal neurons sustained axonal elongation and sprouting. Stretched axons showed typical hallmarks of sustained outgrowth, i.e., low $\mathrm{Ca}^{2+}$ transients, protein increase in the axonal compartment, and ER cisternae accumulation. However, we found a statistically significant increase in MT density (longitudinal cross-section and TEM observation) along the entire axonal shaft from the emergency cone to the GC.

In our opinion, we are still faced with the question of whether MT advances during elongation due to MT translocation or in situ assembly and how force contributes to this.

Our methodology for axonal stretching is based on NP labeling of the entire axonal shaft. We found that NPs accumulated as isolated particles at the axonal membrane and within the axoplasm. TEM imaging showed an intimate contact between NPs and the axon cytoskeleton, and we used magnetic manipulation to generate pushing forces that decreased the net contractile force in the axonal shaft (Eq. 2). However, the forces generated by NPs $(\sim 10 \mathrm{pN})$ would seem to be low compared with the contractile forces generated at the GC and in the axon (O'Toole et al., 2015). However, the effectiveness of extremely low forces as a powerful enhancer of axonal elongation and guidance is beyond any doubt ( $\mathrm{Wu}$ et al., 2012; Kilinc et al., 2014; Pita-Thomas et al., 2015). The increase in MT density that we observed in the axon shaft may indicate that force in itself has a direct effect on the assembly dynamics of MTs.

Given that there are excellent pharmacological tools to interfere with MT stabilization/destabilization, we used our methodology to directly test stretch-growth in presence of nocodazole (MT-destabilizing activity) and paclitaxel (MT-stabilizing activity). We found that nocodazole, but not paclitaxel, differentially impairs stretch-growth versus spontaneous growth, highlighting that stretch-growth cannot occur if MT polymerization is negatively affected by a pharmacologic treatment. The specificity of this mechanism was also confirmed by our use of a myosin-II inhibitor (blebbistatin) and actin polymerization inhibitor (cytochalasin-B) that had no differential effect on stretch-growth versus tip-growth. 
A
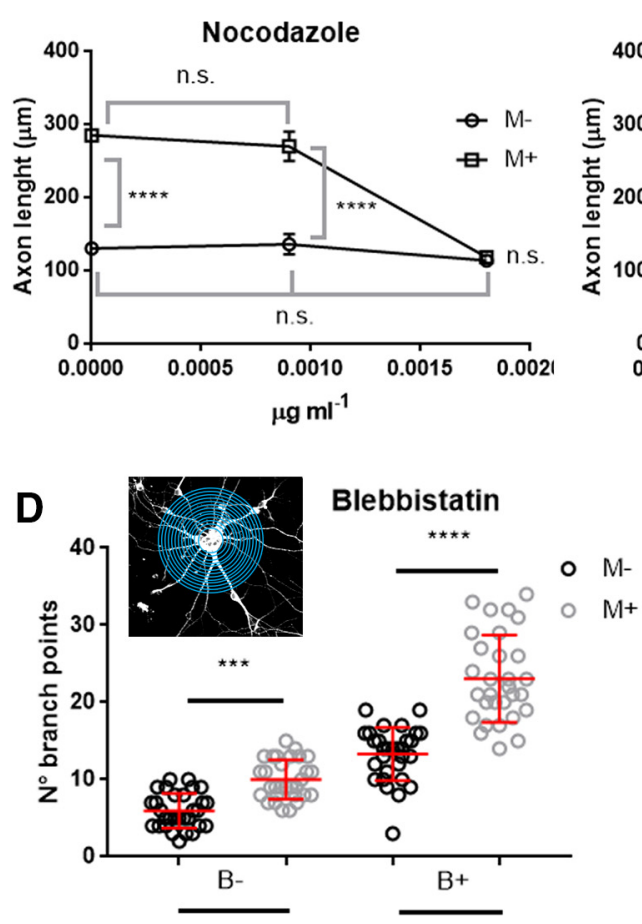

B

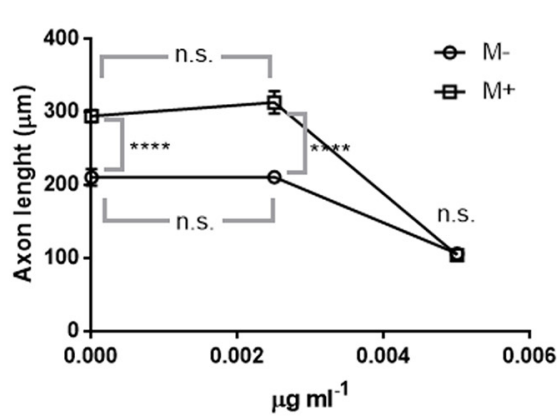

C

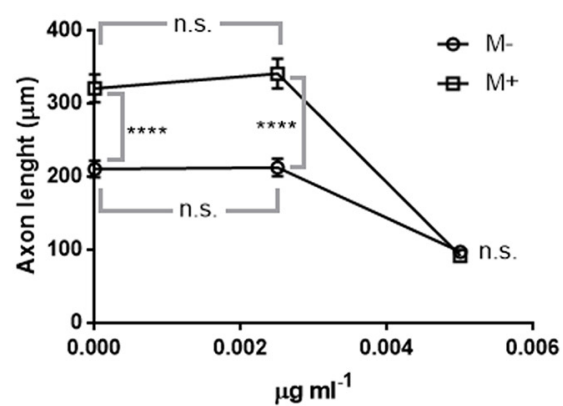

$\mathbf{E}$

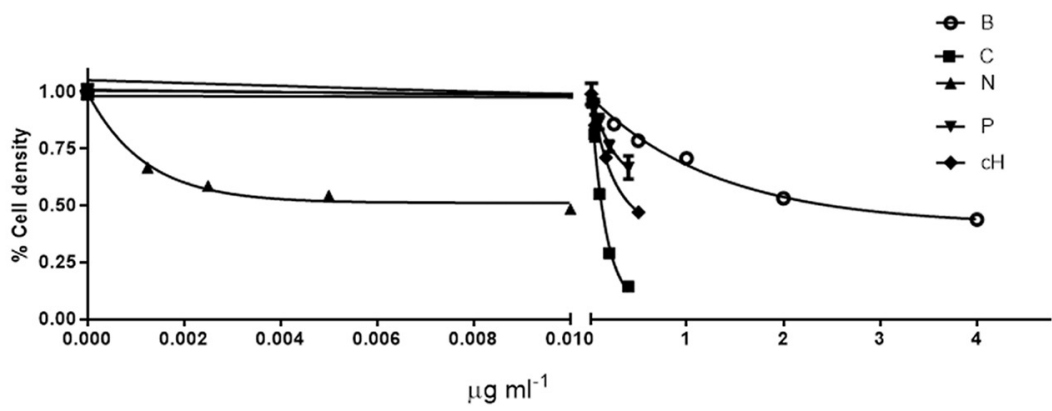

Figure 9. Effects of cytoskeleton component inhibitors. $\boldsymbol{A}$, Nocodazole was added at DIV1 and axon length measured at DIV3 (mean \pm SD). Two-way ANOVA test, with post hoc Tukey's HSD test. Row factor $\left(\mathrm{M}^{+}\right.$vs $\left.\mathrm{M}^{-}\right): p<0.0001, F=99.87$. Columns factor (concentration): $p<0.0001, F=182.4$. B, Paclitaxel was added at DIV1. Axon length at DIV3 (mean \pm SD). Tw0way ANOVA test with post hoc Tukey's HSD test. Row factor $\left(\mathrm{M}^{+}\right.$vs $\left.\mathrm{M}^{-}\right): p<0.0001, F=285.1$. Columns factor (concentration): $p<0.0001, F=77.5$. C, Cytochalasin-B was added at DIV1. Axon length at DIV3 (mean \pm SD). Two-way ANOVA test with post hoc Tukey's HSD test. Row factor $\left(M^{+}\right.$vs $\left.^{-}{ }^{-}\right): p<0.0001, F=315.5$. Columns factor (concentration): $p<0.0001$, $F=97.89 . D, 0.4 \mu \mathrm{g} \mathrm{ml}^{-1} B$ was added at DIV1. Number of branching points at DIV3 (Sholl analysis). Two-way ANOVA test with post hoc Tukey's HSD test. Row factor (B ${ }^{-}$vs B $B^{+}$): $p<0.0001, F=224.2$. Columns factor $\left(M^{-}\right.$vs $\left.M^{+}\right): p<0.0001, F=102.6$. Interaction: $p<0.0001, F=17.71 ; n=30$ neurons from three independent assays, $p<0.0001$. $\boldsymbol{E}$, Toxicity of inhibitors. Dose-response assay: the number of neurons was counted at DIV3; $N=4$, one-phase decay regression analysis. $R^{2}=0.98,0.98,0.96,0.80$, and 0.94 , for $B, C, N, P$, and $C H$, respectively. IC25 $=0.68 \mu \mathrm{g} \mathrm{ml}^{-1}, 0.055 \mu \mathrm{g} \mathrm{ml}^{-1}, 0.9 \mathrm{ng} \mathrm{ml}^{-1}, 0.25 \mu \mathrm{g} \mathrm{ml}^{-1}$, and $0.13 \mu \mathrm{g} \mathrm{ml}^{-1}$ for B, C, N, P, and CH, respectively. Statistical analyses: ${ }^{* * *} p<0.001,{ }^{* * * *} p<0.0001, \mathrm{n}$.S., not statistically significant.

This raises the question as to how force could influence MT assembly. The possibility that MTs are tension sensors has been recently explored (Hamant et al., 2019). The application of a tensile force can induce randomly moving MTs to align themselves along the tension lines in vitro (Kabir et al., 2014). While a compressional force reduces the growth velocity of MTs (Janson et al., 2003), the application of a tensile force to the plus end of MT was found to accelerate MT growth in an in vitro assay of MT polymerization (Franck et al., 2007; Trushko et al., 2013). For example, a force increasing from 0.5 to $2 \mathrm{pN}$ was found to double MT growth. However, how stress is sensed by MT is a question that still needs to be addressed in future work.

In conclusion, our experimental evidence supports the idea that the application of extremely low forces could contribute directly or indirectly both to MT translocation and assembly.

Our findings could support the development of novel strategies for axonal regeneration. NP-mediated manipulation could stimulate neurite guidance (Riggio et al., 2014), axonal outgrowth, and neuron maturation. We found that stretch-growth stimulates the maturation of synapses, with an early presynaptic increase in the frequency of glutamatergic currents, which is then accompanied by a postsynaptic enhancement in neurotransmitter release, in addition to enhancing neuronal excitability. Forces are among the most effective known stimuli for promoting axonal elongation (Franze et al., 2013). They can also act as downstream effectors of many signaling cascades activated by neurotrophins (Myers and Gomez, 2011; Toriyama et al., 2013; Turney et al., 2016). This is also highlighted by the fact that we found that there was a loss of responsiveness to BDNF of stretched axons, which suggests an interference between the two pathways or a saturation effect of the same downstream pathway. Revealing the molecular network responsible for stretch-growth will be the focus of our future research, which we believe holds a promising potential for regenerative medicine.

\section{References}

Athamneh AIM, He Y, Lamoureux P, Fix L, Suter DM, Miller KE (2017) Neurite elongation is highly correlated with bulk forward translocation of microtubules. Sci Rep 7:7292.

Bullivant JP, Zhao S, Willenberg BJ, Kozissnik B, Batich CD, Dobson J (2013) Materials characterization of feraheme/ferumoxytol and preliminary evaluation of its potential for magnetic fluid hyperthermia. Int J Mol Sci 14:17501-17510.

Calatayud MP, Riggio C, Raffa V, Sanz B, Torres TE, Ibarra MR, Hoskins C, Cuschieri A, Wang L, Pinkernelle J, Keilhoff G, Goya GF (2013) Neuronal cells loaded with PEI-coated Fe3O4 nanoparticles for magnetically guided nerve regeneration. J Mater Chem B 1:3607-3616.

Convertino D, Fabbri F, Mishra N, Mainardi M, Cappello V, Signore G, Testa G, Capsoni S, Albertazzi L, Cattaneo A, Luin S, Marchetti L, Coletti C (2013) Graphene promotes axon elongation through local stall of Nerve Growth Factor signaling endosomes. Nano Lett 20:3633-3641.

Franck AD, Powers AF, Gestaut DR, Gonen T, Davis TN, Asbury CL (2007) Tension applied through the Dam1 complex promotes microtubule elongation providing a direct mechanism for length control in mitosis. Nat Cell Biol 9:832-837. 
Franze K (2013) The mechanical control of nervous system development. Development 140:3069-3077.

Franze K, Guck J (2010) The biophysics of neuronal growth. Rep Prog Phys 73:094601.

Franze K, Janmey PA, Guck J (2013) Mechanics in neuronal development and repair. Annu Rev Biomed Eng 15:227-251.

Hamant O, Inoue D, Bouchez D, Dumais J, Mjolsness E (2019) Are microtubules tension sensors?. Nat Commun 10:2360.

Janson ME, de Dood ME, Dogterom M (2003) Dynamic instability of microtubules is regulated by force. J Cell Biol 161:1029-1034.

Kabir AMR, Inoue D, Hamano Y, Mayama H, Sada K, Kakugo A (2014) Biomolecular motor modulates mechanical property of microtubule. Biomacromolecules 15:1797-1805.

Kilinc D, Blasiak A, O'Mahony JJ, Lee GU (2014) Low piconewton towing of CNS axons against diffusing and surface-bound repellents requires the inhibition of motor protein-associated pathways. Sci Rep 4:7128.

Kollins KM, Hu J, Bridgman PC, Huang YQ, Gallo G (2009) Myosin-II negatively regulates minor process extension and the temporal development of neuronal polarity. Dev Neurobiol 69:279-298.

Koser DE, Thompson AJ, Foster SK, Dwivedy A, Pillai EK, Sheridan GK, Svoboda H, Viana M, Costa LdF, Guck J, Holt CE, Franze K (2016) Mechanosensing is critical for axon growth in the developing brain. Nat Neurosci 19:1592-1598.

Kutzing MK, Langhammer CG, Luo V, Lakdawala H, Firestein BL (2010) Automated Sholl analysis of digitized neuronal morphology at multiple scales. J Vis Exp. Advance online publication. Retrieved November 14, 2010. doi: $10.3791 / 2354$.

Labelle C, Leclerc N (2000) Exogenous BDNF, NT-3 and NT-4 differentially regulate neurite outgrowth in cultured hippocampal neurons. Brain Res Dev Brain Res 123:1-11.

Lamoureux P, Heidemann SR, Martzke NR, Miller KE (2010) Growth and elongation within and along the axon. Dev Neurobiol 70:135-149.

Lin AC, Holt CE (2007) Local translation and directional steering in axons. EMBO J 26:3729-3736.

Lu W, Fox P, Lakonishok M, Davidson MW, Gelfand VI (2013) Initial neurite outgrowth in Drosophila neurons is driven by kinesin-powered microtubule sliding. Curr Biol 23:1018-1023.

Miller KE, Suter DM (2018) An integrated cytoskeletal model of neurite outgrowth. Front Cell Neurosci 12:447.

Mutalik SP, Joseph J, Pullarkat PA, Ghose A (2018) Cytoskeletal mechanisms of axonal contractility. Biophys J 115:713-724.

Myers JP, Gomez TM (2011) Focal adhesion kinase promotes integrin adhesion dynamics necessary for chemotropic turning of nerve growth cones. J Neurosci 31:13585-13595.

O'Toole M, Lamoureux P, Miller KE (2008) A physical model of axonal elongation: force, viscosity, and adhesions govern the mode of outgrowth. Biophys J 94:2610-2620.

O'Toole M, Lamoureux P, Miller KE (2015) Measurement of subcellular force generation in neurons. Biophys J 108:1027-1037.

Oelz DB, Del Castillo U, Gelfand VI, Mogilner A (2018) Microtubule dynamics, kinesin-1 sliding, and dynein action drive growth of cell processes. Biophys J 115:1614-1624.

Pita-Thomas W, Steketee MB, Moysidis SN, Thakor K, Hampton B, Goldberg JL (2015) Promoting filopodial elongation in neurons by membrane-bound magnetic nanoparticles. Nanomedicine 11:559-567.

Popko J, Fernandes A, Brites D, Lanier LM (2009) Automated analysis of NeuronJ tracing data. Cytometry A 75:371-376.

Raffa V, Falcone F, De Vincentiis S, Falconieri A, Calatayud MP, Goya GF, Cuschieri A (2018) Piconewton mechanical forces promote neurite growth. Biophys J 115:2026-2033.
Rao AN, Patil A, Black MM, Craig EM, Myers KA, Yeung HT, Baas PW (2017) Cytoplasmic dynein transports axonal microtubules in a polaritysorting m. Cell Rep 19:2210-2219.

Riggio C, Calatayud MP, Giannaccini M, Sanz B, Torres TE, FernándezPacheco R, Ripoli A, Ibarra MR, Dente L, Cuschieri A, Goya GF, Raffa V (2014) The orientation of the neuronal growth process can be directed via magnetic nanoparticles under an applied magnetic field. Nanomedicine 10:1549-1558.

Roossien DH, Lamoureux P, Van Vactor D, Miller KE (2013) Drosophila growth cones advance by forward translocation of the neuronal cytoskeletal meshwork in vivo. PLoS One 8:e80136.

Roossien DH, Lamoureux P, Miller KE (2014) Cytoplasmic dynein pushes the cytoskeletal meshwork forward during axonal elongation. J Cell Sci 127:3593-3602.

Rosenberg SS, Spitzer NC (2011) Calcium signaling in neuronal development. Cold Spring Harb Perspect Biol 3:a004259.

Ruthel G, Hollenbeck PJ (2000) Growth cones are not required for initial establishment of polarity or differential axon branch growth in cultured hippocampal neurons. J Neurosci 20:2266-2274.

Siano G, Varisco M, Caiazza MC, Quercioli V, Mainardi M, Ippolito C, Cattaneo A, Di Primio C (2019) Tau modulates VGluT1 expression. J Mol Biol 431:873-884.

Smith DM (2009) Stretch growth of integrated axon tracts: Extremes and exploitations. Prog Neurobiol 89:231-239.

Suter DM, Miller KE (2011) The emerging role of forces in axonal elongation. Prog Neurobiol 94:91-101.

Testa G, Olimpico F, Pancrazi L, Borello U, Cattaneo A, Caleo M, Costa M, Mainardi M (2019) Cortical seizures in FoxG1(+/-) mice are accompanied by Akt/S6 overactivation, excitation/inhibition imbalance and impaired synaptic transmission. Int J Mol Sci 20:4127.

Toriyama M, Kozawa S, Sakumura Y, Inagaki N (2013) Conversion of a signal into forces for axon outgrowth through Pak1-mediated shootin1 phosphorylation. Curr Biol 23:529-534.

Trushko A, Schäffer E, Howard J (2013) The growth speed of microtubules with XMAP215-coated beads coupled to their ends is increased by tensile force. Proc Natl Acad Sci USA 110:14670-14675.

Turney SG, Ahmed M, Chandrasekar I, Wysolmerski RB, Goeckeler ZM, Rioux RM, Whitesides GM, Bridgman PC (2016) Nerve growth factor stimulates axon outgrowth through negative regulation of growth cone actomyosin restraint of microtubule advance. Mol Biol Cell 27:500-517.

Valenzuela JI, Perez F (2015) Diversifying the secretory routes in neurons. Front Neurosci 9:358.

van Horck FP, Weinl C, Holt CE (2004) Retinal axon guidance: novel mechanisms for steering. Curr Opin Neurobiol 14:61-66.

Voelzmann A, Hahn I, Pearce SP, Sánchez-Soriano N, Prokop A (2016) A conceptual view at microtubule plus end dynamics in neuronal axons. Brain Res Bull 126:226-237.

Woo S, Gomez TM (2006) Racl and RhoA promote neurite outgrowth through formation and stabilization of growth cone point contacts. J Neurosci 26:1418-1428.

Wu T, Nieminen TA, Mohanty S, Miotke J, Meyer RL, Rubinsztein-Dunlop H, Berns MW (2012) A photon-driven micromotor can direct nerve fibre growth. Nature Photon 6:62-67.

Yu W, Baas PW (1994) Changes in microtubule number and length during axon differentiation. J Neurosci 14:2818-2829.

Zheng J, Lamoureux P, Santiago V, Dennerll T, Buxbaum RE, Heidemann SR (1991) Tensile regulation of axonal elongation and initiation. J Neurosci 11:1117-1125. 